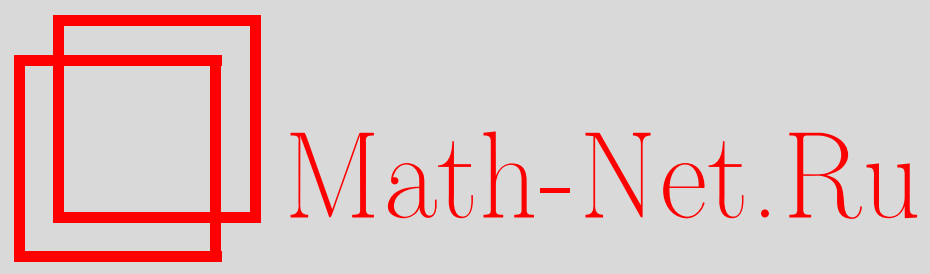

И. Б. Кожухов, В. А. Ярошевич, О потенциальной делимости матриц над дистрибутивными решетками, Дискрет. матем., 2010, том 22, выпуск 2, 148-159

DOI: https://doi.org/10.4213/dm1101

Использование Общероссийского математического портала Math-Net.Ru подразумевает, что вы прочитали и согласны с пользовательским соглашением http://www . mathnet.ru/rus/agreement

Параметры загрузки:

IP : 18.234 .197 .8

26 апреля 2023 г., 12:46:14 


\title{
О потенциальной делимости матриц над дистрибутивными решетками
}

\author{
() 2010 г. И. Б. Кожухов, В. А. Ярошевич
}

\begin{abstract}
Рассматриваются матрицы произвольных размеров (в том числе бесконечных) над дистрибутивной решеткой $L$. Доказано, что если $L=2^{X}-$ решетка подмножеств множества $X$, то потенциальная делимость матриц (слева или справа) друг на друга равносильна обычной делимости. В частности, в полугруппе квадратных матриц над решеткой $2^{X}$ отношение Грина $\mathscr{L}$ совпадает с обобщенным отношением Грина $\mathscr{L}^{*}$
\end{abstract}

Пусть $S$ - полугруппа и $a, b \in S$. Говорят, что элемент $b$ делится на $a$ слева, если $b=s a$ при некотором $s \in S^{1}$, где $S^{1}=S \cup\{1\}$. Элемент $b$ делится слева на $a$ потенциально, если $b=t a$ в некоторой надполугруппе $T \supseteq S, t \in T$. Известно, что $b$ делится на $a$ слева потенциально в том и только в том случае, если выполняется условие

$$
\forall x, y \in S^{1} \quad(a x=a y \Longrightarrow b x=b y)
$$

(см. гл.10 в [1] и [2]). Интересным является вопрос, какие полугруппы могут быть взяты в качестве надполугрупп $T$. В $[1,2]$ (см. также теоремы 7, 11 в [3]) в качестве полугруппы $T \supseteq S$ была взята полугруппа $\mathscr{T}\left(S^{1}\right)$ всех преобразований множества $S^{1}$. Оказывается в качестве полугруппы $T$ может быть взята любая полугруппа $T \supseteq S$, в которой отношение левой делимости совпадает с отношением потенциальной левой делимости (например, любая регулярная полугруппа). Далее мы изучаем отношение делимости друг на друга матриц над дистрибутивными решетками и отношение потенциальной делимости. При этом матрицы необязательно квадратные, а в случае, когда решетка имеет точные верхние грани любых подмножеств (не только конечных), можно рассматривать и бесконечные матрицы. Доказано, что для широкого класса дистрибутивных решеток $L$ потенциальная делимость матриц над $L$ равносильна обычной делимости над некоторой надрешеткой $\widetilde{L} \supseteq L$. Если рассматриваются лишь матрицы конечных размеров, то достаточно дистрибутивности решетки $L$. Эти результаты мы применяем затем к исследованию отношений Грина $\mathscr{L}, \mathscr{R}$ на полугруппах матриц над дистрибутивными решетками и обобщенных отношений Грина $\mathscr{L}^{*}, \mathscr{R}^{*}$ на этих полугруппах. В частности, показано, что если $L$ изоморфна решетке всех подмножеств некоторого множества, то в полугруппе квадратных матриц над $L$ справедливо равенство $\mathscr{L}=\mathscr{L}^{*}$. Следовательно, $\mathscr{L}=\mathscr{L}^{*}$ в полугруппе бинарных отношений, так как эта полугруппа изоморфна полугруппе квадратных матриц одного размера над решеткой $L=\{0,1\}$. Доказано, что необходимым условием равенства $\mathscr{L}=\mathscr{L}^{*}$ в полугруппе матриц над $L$ является наличие в решетке $L$ относительных дополнений у каждого элемента.

Основные сведения из теории полугрупп можно найти в $[1,4]$, из теории решеток - в [5]. Матрицы над дистрибутивными решетками изучались во многих работах, обзор 
результатов которых не является целью данной статьи. Упомянем лишь известную монографию [6], в которой обстоятельно изложена теория булевых матриц. Отношения Грина для матриц над булевыми алгебрами и их связь с рангами матриц изучались в [7].

Пусть $S$ - полугруппа и $a, b \in S$. Отношение левой делимости будем обозначать через $\leqslant l$, а потенциальной левой делимости - через $\leqslant_{l}^{*}$, то есть

$$
\begin{aligned}
& b \leqslant_{l} a \Longleftrightarrow b=s a \text { при некотором } s \in S^{1}, \\
& b \leqslant_{l}^{*} a \Longleftrightarrow b=t a \text { при некотором } t \in T,
\end{aligned}
$$

где $T \supseteq S-$ некоторая надполугруппа полугруппы $S$.

Теорема 1. Пусть $S-$ полугруппа, $T \supseteq S-$ такая ее надполугруппа, в которой отношения $\leqslant_{l} u \leqslant_{l}^{*}$ совпадают. Тогда для любых $a, b \in S$

$$
b \leqslant_{l}^{*} a \Longleftrightarrow b \in T^{1} a .
$$

Доказательство. Импликация $\Leftarrow$ очевидна. Докажем импликацию $\Rightarrow$. Пусть $b \leqslant{ }_{l}^{*} a$. Через $S^{1}$ будем обозначать полугруппу, полученную присоединением к $S$ единицы, даже если в $S$ уже была единица: $S^{1}=S \cup\{1\}, 1 \notin S$. Аналогично $T^{1}=T \cup\{1\}, 1 \notin T$. Кроме того, будем считать, что единицы в $S^{1}$ и $T^{1}$ совпадают. Обозначим через $\mathscr{P} \mathscr{T}(X)$ полугруппу частичных отображений $X_{1} \rightarrow X_{2}$, где $X_{1}, X_{2} \subseteq X$, с умножением $(\alpha \beta)(x)=\alpha(\beta(x))$. При этом $\operatorname{dom}(\alpha \beta)=\operatorname{dom} \beta \cap \beta^{-1}(\operatorname{dom} \alpha)$, где $\operatorname{dom}$ обозначает область определения. К естественным вложениям $S \rightarrow T \rightarrow T^{1}$ добавим вложение $T^{1} \rightarrow \mathscr{P} \mathscr{T}\left(T^{1}\right) u \mapsto \varphi_{u}$, где $\operatorname{dom} \varphi_{u}=S^{1}$ и $\varphi_{u}(x)=u x$ при $x \in S^{1}$. Так как $b \leqslant_{l}^{*} a$ в $S,(a x=a y \Longrightarrow b x=b y)$ для всех $x, y \in S^{1}$. Построим отображение $\psi: a S^{1} \rightarrow b S^{1}$, полагая $\psi(a x)=b x$ при $x \in S^{1}$. Ввиду выполнимости условия (1) отображение $\psi$ определено корректно и $\psi \in \mathscr{P} \mathscr{T}\left(T^{1}\right)$. Ясно, что $\psi \varphi_{a}=\varphi_{b}$. Отождествляя $a$ с $\varphi_{a}, b$ с $\varphi_{b}$, получим, что $b \in \mathscr{P} \mathscr{T}\left(T^{1}\right) a$. Это означает, что $b \leqslant_{l}^{*} a$ в полугруппе $T$. Так как в полугруппе $T$ по условию отношения $\leqslant l$ и $\leqslant_{l}^{*}$ совпадают, справедливо включение $b \in T^{1} a$.

Отношения Грина $\mathscr{L}, \mathscr{R}$ на полугруппе $S$ определяются следующим образом (см. гл. 2 в [4]):

$$
\begin{aligned}
& a \mathscr{L} b \Longleftrightarrow S^{1} a=S^{1} b, \\
& a \mathscr{R} b \Longleftrightarrow a S^{1}=b S^{1} .
\end{aligned}
$$

Очевидно, $\mathscr{L}=\leqslant_{l} \cap\left(\leqslant_{l}\right)^{-1}$. Обобщенные отношения Грина $\mathscr{L}^{*}, \mathscr{R}^{*}-$ это потенциальные расширения отношений $\mathscr{L}, \mathscr{R}$, а именно,

$$
\begin{aligned}
& a \mathscr{L}^{*} b \text { в } S \Longleftrightarrow a \mathscr{L} b \text { в некоторой надполугруппе } T \supseteq S, \\
& a \mathscr{R}^{*} b \text { в } S \Longleftrightarrow a \mathscr{R} b \text { в некоторой надполугруппе } T \supseteq S
\end{aligned}
$$

(см. [8]). Таким образом,

$$
\begin{aligned}
& a \mathscr{L}^{*} b \Longleftrightarrow \forall x, y \in S^{1} \quad(a x=a y \Longleftrightarrow b x=b y), \\
& a \mathscr{R}^{*} b \Longleftrightarrow \forall x, y \in S^{1} \quad(x a=y a \Longleftrightarrow x b=y b)
\end{aligned}
$$

и $\mathscr{L}^{*}=\leqslant_{l}^{*} \cap\left(\leqslant_{l}^{*}\right)^{-1}$.

Заметим, что если $a$ - регулярный элемент полугруппы $S$ (то есть $a \in a S a$ ) и $b \leqslant_{l}^{*} a$, то $b \leqslant_{l} a$. Действительно, $a=a s a$ при некотором $s \in S$, то есть $a \cdot 1=a \cdot s a$, что влечет равенство $b \cdot 1=b \cdot s a$, откуда $b \in S a$. Из замечания следует, что в регулярной полугруппе отношения $\leqslant_{l}^{*}$ и $\leqslant l$ совпадают, а значит, $\mathscr{L}=\mathscr{L}^{*}$. 
Теорема 2. Пусть $S$ - подполугруппа полугруппь $T$ и в полугруппе $T$ имеет место равенство $\mathscr{L}=\mathscr{L}^{*}$. Тогда для элементов $a, b \in S$

$$
(a, b) \in \mathscr{L}^{*} \Longleftrightarrow(a, b) \in \mathscr{L} \text { в } T \text {. }
$$

Доказательство. Импликация $\Leftarrow$ очевидна. Докажем, что верна импликация $\Rightarrow$. Пусть $(a, b) \in \mathscr{L}^{*}$. Вложим $T$ в регулярную полугруппу $A$ (например, в качестве $A$ можно взять полугруппу $\left.\mathscr{T}\left(T^{1}\right)\right)$. Так как $(a, b) \in \mathscr{L}^{*}$, справедливы соотношения $a \leqslant_{l}^{*} b$ и $b \leqslant_{l}^{*} a$ в $S$. По теореме $1 a \leqslant_{l} b$ и $b \leqslant_{l} a$ в полугруппе $A$. Это означает, что $a \leqslant_{l}^{*} b$ и $b \leqslant_{l}^{*} a$ в полугруппе $T$, то есть $(a, b) \in \mathscr{L}^{*}$ в $T$. В полугруппе $T$ по условию $\mathscr{L}=\mathscr{L}^{*}$, поэтому $(a, b) \in \mathscr{L}$ в $T$.

Перейдем теперь к матрицам над дистрибутивными решетками. Мы будем рассматривать матрицы $\sigma=\left\|\sigma_{i j}\right\|_{i \in I, j \in J}$ над дистрибутивной решеткой $L$, то есть $\sigma_{i j} \in L$. Так как матрицы могут быть бесконечными, будем предполагать, что в решетке $L$ любое подмножество $\left\{x_{i} \mid i \in I\right\}$ имеет точную верхнюю грань $\bigvee_{i \in I} x_{i}$ и выполнен закон бесконечной дистрибутивности

$$
y \wedge \bigvee_{i} x_{i}=\bigvee_{i}\left(y \wedge x_{i}\right)
$$

Наличие точной нижней грани бесконечного подмножества и выполнение другого закона бесконечной дистрибутивности (двойственного вышеприведенному), вообще говоря, не требуется.

Умножение матриц $\sigma=\left\|\sigma_{i j}\right\|_{i \in I, j \in J}$ и $\tau=\left\|\tau_{j k}\right\|_{j \in J, k \in K}$ осуществляется по правилу

$$
\sigma \tau=\rho=\left\|\rho_{i k}\right\|_{i \in I, k \in K},
$$

где

$$
\rho_{i k}=\bigvee_{j \in J}\left(\sigma_{i j} \wedge \tau_{j k}\right) .
$$

Матрицы необязательно квадратные, то есть мощности множеств $I, J, K$ могут быть различными. Матрица $I \times 1$ называется строкой, а матрица $1 \times I-$ столбцом. Операции на матрицах одного размера определяются покомпонентно: если $a=\left\|a_{i j}\right\|, b=\left\|b_{i j}\right\|$, то

$$
\begin{aligned}
& a \wedge b=\left\|a_{i j} \wedge b_{i j}\right\|, \\
& a \vee b=\left\|a_{i j} \vee b_{i j}\right\|, \\
& \lambda \wedge a=\left\|\lambda \wedge a_{i j}\right\|, \quad \lambda \in L .
\end{aligned}
$$

Полагаем $a \leqslant b$, если $a_{i j} \leqslant b_{i j}$ для любых $i \in I, j \in J$. Линейной комбинацией строк $a^{(\gamma)}, \gamma \in \Gamma$, называется строка $\bigvee_{\gamma \in \Gamma}\left(\lambda^{(\gamma)} \wedge a^{(\gamma)}\right)$, где $\lambda^{(\gamma)} \in L$ при $\gamma \in \Gamma$. Если $M=\left\{a^{(\gamma)} \mid \gamma \in \Gamma\right\}-$ множество строк, то множество всех линейных комбинаций этих строк называется линейной оболочкой множества $M$ и обозначается $\operatorname{Span} M$.

Лемма 1. Пусть $M=\left\{a^{(\gamma)} \mid \gamma \in \Gamma\right\}$-множество строк $a^{(\gamma)}=\left\|a_{i}^{(\gamma)}\right\|_{i \in I}$ u $b=\left\|b_{i}\right\|_{i \in I}$ - строка того же размера. Положим

$$
\mu^{(\gamma)}=\bigvee\left\{x \mid x \wedge a^{(\gamma)} \leqslant b\right\} .
$$

Тогда

$$
b \in \operatorname{Span} M \Longleftrightarrow b=\bigvee_{\gamma \in \Gamma}\left(\mu^{(\gamma)} \wedge a^{(\gamma)}\right)
$$


Доказательство. Докажем, что верна импликация $\Rightarrow$. Если $b \in \operatorname{Span} M$, то

$$
b=\bigvee_{\gamma \in \Gamma}\left(\lambda^{(\gamma)} \wedge a^{(\gamma)}\right)
$$

при некоторых $\lambda^{\gamma} \in L$. Отсюда

$$
b_{i}=\bigvee_{\gamma}\left(\lambda^{(\gamma)} \wedge a_{i}^{(\gamma)}\right)
$$

при всех $i \in I$. Из (1) получаем, что $\lambda^{(\gamma)} \leqslant \mu^{(\gamma)}$. Справедливы соотношения

$$
b=\bigvee_{\gamma}\left(\lambda^{(\gamma)} \wedge a^{(\gamma)}\right) \leqslant \bigvee_{\gamma}\left(\mu^{(\gamma)} \wedge a^{(\gamma)}\right) \leqslant b
$$

Таким образом,

$$
b=\bigvee_{\gamma}\left(\mu^{(\gamma)} \wedge a^{(\gamma)}\right) .
$$

Импликация $\Leftarrow$ очевидна.

Пусть $\sigma$ и $\tau-J \times K$ и $I \times K$ матрицы соответственно с элементами из $L$. Делимость слева $\tau$ на $\sigma$ означает существование такой $I \times J$ матрицы $\rho$ над $L$, что $\tau=\rho \sigma$. Из определения произведения матриц видно, что делимость $\tau$ на $\sigma$ имеет место в том и только в том случае, когда каждая строка матрицы $\tau$ является линейной комбинацией строк матрицы $\sigma$ (как в случае матриц над кольцом или полукольцом).

Лемма 2. Пусть $\sigma u \tau-J \times K u I \times K$ матрищь соответственно над дистрибутивной решеткой $L$, в которой выполнено тождество (4). Для $i \in I, j \in J$ положим

$$
\mu_{i j}=\bigvee\left\{x \mid \forall k \in K x \wedge \sigma_{j k} \leqslant \tau_{i k}\right\}, \quad \mu=\left\|\mu_{i j}\right\|_{i \in I, j \in J} .
$$

Если $\tau=\rho \sigma$ для некоторой матрицьы $\rho$, то $\tau=\mu \sigma$.

Доказательство. По лемме 1

$$
\tau_{i k}=\bigvee_{j \in J}\left(\mu_{i j} \wedge \sigma_{j k}\right)
$$

откуда следует, что $\tau=\mu \sigma$.

Замечание 1. Матрица $\mu$, построенная в лемме 2, является наибольшей из всех матриц $\rho$, удовлетворяющих равенству $\tau=\mu \sigma$, то есть

$$
\forall \rho \quad(\tau=\rho \sigma \Longrightarrow \mu \geqslant \rho) .
$$

Пусть $L$ - дистрибутивная решетка с нулем, в которой выполнено условие (4), $b=\left\|b_{i}\right\|_{i \in I}, a^{(\gamma)}=\left\|a_{i}^{(\gamma)}\right\|_{i \in I}, \gamma \in \Gamma$, - строки над $L$. Рассмотрим следующее условие на строки $b, a^{(\gamma)}$ : для всех $\rho, \rho^{\prime}$

$$
\left(\left(\forall \gamma \quad a^{(\gamma)} \rho=a^{(\gamma)} \rho^{\prime}\right) \Longrightarrow b \rho=b \rho^{\prime}\right),
$$

где $\rho=\left\|\rho_{i}\right\|_{i \in I}$ и $\rho^{\prime}=\left\|\rho_{i}^{\prime}\right\|_{i \in I}-$ столбцы.

Заметим, что если $L-$ решетка с нулем, в которой существуют точные верхние грани любого подмножества, то в ней существуют и точные нижние грани любого подмножества, то есть $L-$ полная решетка. 
Предложение 1. Пусть $L-$ полная дистрибутивная решетка, в которой выполнено тождество (4) $и b=\left\|b_{i}\right\|_{i \in I}, a^{(\gamma)}=\left\|a_{i}^{(\gamma)}\right\|_{i \in I}, \gamma \in \Gamma,-$ строки с элементами из $L$. тогда

(1) если $b \in \operatorname{Span}\left\{a^{(\gamma)} \mid \gamma \in \Gamma\right\}$, то выполняется условие (6);

(2) если выполнено условие (6), то

$$
b \leqslant \bigvee\left\{a^{(\gamma)} \mid \gamma \in \Gamma\right\} .
$$

Доказательство. Докажем п. 1. Пусть

$$
b=\bigvee_{\gamma}\left(\lambda^{(\gamma)} \wedge a^{(\gamma)}\right), \quad a^{(\gamma)} \rho=a^{(\gamma)} \rho^{\prime}
$$

при всех $\gamma \in \Gamma$. Тогда

$$
\begin{aligned}
b \rho & =\bigvee_{i}\left(b_{i} \wedge \rho_{i}\right)=\bigvee_{i}\left(\bigvee_{\gamma}\left(\lambda^{(\gamma)} \wedge a_{i}^{(\gamma)}\right) \wedge \rho_{i}\right)=\bigvee_{i} \bigvee_{\gamma}\left(\lambda^{(\gamma)} \wedge a_{i}^{(\gamma)} \wedge \rho_{i}\right) \\
& =\bigvee_{\gamma} \bigvee_{i}\left(\lambda^{(\gamma)} \wedge a_{i}^{(\gamma)} \wedge \rho_{i}\right)=\bigvee_{\gamma}\left(\lambda^{(\gamma)} \wedge \bigvee_{i}\left(a_{i}^{(\gamma)} \wedge \rho_{i}\right)\right) \\
& =\bigvee_{\gamma}\left(\lambda^{(\gamma)} \wedge \bigvee_{i}\left(a_{i}^{(\gamma)} \wedge \rho_{i}^{\prime}\right)\right)=b \rho^{\prime},
\end{aligned}
$$

то есть выполнено условие (6).

Докажем п. 2. Пусть условие (7) не выполнено. Тогда

$$
b_{i_{0}} \not \bigvee_{\gamma \in \Gamma} a_{i_{0}}^{(\gamma)}
$$

при некотором $i_{0} \in I$. Положим

$$
c_{i_{0}}=\bigvee_{\gamma} a_{i_{0}}^{(\gamma)}
$$

Очевидно, $b_{i_{0}} \wedge c_{i_{0}}<b_{i_{0}}$. Положим

$$
\rho_{i}=\left\{\begin{array}{ll}
0 & \text { при } i \neq i_{0}, \\
1 & \text { при } i=i_{0},
\end{array} \quad \rho_{i}^{\prime}=\left\{\begin{array}{ll}
0 & \text { при } i \neq i_{0}, \\
c_{i_{0}} & \text { при } i=i_{0} .
\end{array} .\right.\right.
$$

Справедливы равенства

$$
\begin{aligned}
a^{(\gamma)} \rho & =a_{i_{0}}^{(\gamma)} \wedge \rho_{i_{0}}=a_{i_{0}}^{(\gamma)}, a^{(\gamma)} \rho^{\prime}=a_{i_{0}}^{(\gamma)} \wedge \rho_{i_{0}}^{\prime}=a_{i_{0}}^{(\gamma)} \wedge \bigvee_{\delta} a_{i_{0}}^{(\delta)} \\
& =a_{i_{0}}^{(\gamma)}, b \rho=b_{i_{0}} \wedge \rho_{i_{0}}=b_{i_{0}}, b \rho^{\prime}=b_{i_{0}} \wedge \rho_{i_{0}}^{\prime}=b_{i_{0}} \wedge c_{i_{0}}<b_{i_{0}} .
\end{aligned}
$$

Так как $b \rho \neq b \rho^{\prime}$, условие (6) не выполнено.

В общем случае условие (6) не гарантирует, что $b \in \operatorname{Span}\left\{a^{(\gamma)} \mid \gamma \in \Gamma\right\}$. Приведем соответствующий пример. 
Пример 1. Пусть $L=\{0, p, 1 \mid 0<p<1\}-$ трехэлементная цепь, $b=(1,0)$, $a^{(1)}=(p, 0), a^{(2)}=(1, p)$. Нетрудно проверить, что для этих строк выполнено условие (6). Однако строка $b$ не является линейной комбинацией строк $a^{(1)}, a^{(2)}$. Проверим это. По лемме 1 , если $b \in \operatorname{Span}\left(a^{(1)}, a^{(2)}\right)$, то

$$
b=\left(\mu^{(1)} \wedge a^{(1)}\right) \vee\left(\mu^{(2)} \wedge a^{(2)}\right),
$$

где

$$
\mu^{(1)}=\bigvee\{x \mid x \wedge p \leqslant 1, x \wedge 0 \leqslant 0\}, \quad \mu^{(2)}=\bigvee\{y \mid y \wedge 1 \leqslant 1, y \wedge p \leqslant 0\}
$$

Ясно, что $\mu^{(1)}=1, \mu^{(2)}=0$, но

$$
\left(\mu^{(1)} \wedge a^{(1)}\right) \vee\left(\mu^{(2)} \wedge a^{(2)}\right)=(1 \wedge(p, 0)) \vee(0 \wedge(1, p))=(p, 0) \neq b .
$$

Хотя $b$ не является линейной комбинацией строк $a^{(1)}, a^{(2)}$ с коэффициентами из $L$, но решетку $L$ можно расширить до решетки $\widetilde{L}$ так, что строка $b$ будет являться линейной комбинаций строк с коэффициентами из $\tilde{L}$. Действительно, пусть

$$
\widetilde{L}=\{0, p, q, 1 \mid 0<p<1,0<q<1\} .
$$

Очевидно, $q$ является дополнением в $\widetilde{L}$ элемента $p: p \wedge q=0, p \vee q=1$. Так как

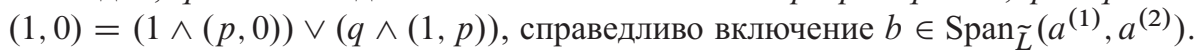

Построенный выше пример инициирует введение следующего определения. Пусть $b=\left\|b_{i}\right\|_{i \in I}$ и $a^{(\gamma)}=\left\|a_{i}^{(\gamma)}\right\|_{i \in I}, \gamma \in \Gamma,-$ строки с элементами из $L$. Будем говорить, что строка $b$ является линейной комбинацией строк $a^{(\gamma)}, \gamma \in \Gamma$, в потенциальном смысле, если существует такая надрешетка $\widetilde{L} \supseteq L$, что

$$
b=\bigvee_{\gamma \in \Gamma}\left(\xi^{(\gamma)} \wedge a^{(\gamma)}\right)
$$

для некоторых $\xi^{(\gamma)} \in \widetilde{L}$. Часть 1 предложения 1 может быть усилена. А именно, справедливо следующее утверждение.

Предложение 2. Если строка $b=\left\|b_{i}\right\|_{i \in I}$ является линейной комбинацией строк $a^{(\gamma)}=\left\|a_{i}^{(\gamma)}\right\|_{i \in I}$ в потенциильном смысле, то для этих строк выполнено условие (6).

Доказательство не отличается от доказательства утверждения 1 предложения 1.

Интересен вопрос о возможности обращения предложения 2, а именно, верно ли, что из условия (6) следует, что строка $b$ является линейной комбинацией в потенциальном смысле строк $a^{(\gamma)}, \gamma \in \Gamma$. Мы не знаем ответа на этот вопрос в общем случае. Ниже будет доказано, что ответ положителен для решеток $L$, имеющих полное вложение в решетку $2^{X}$ всех подмножеств какого-либо множества $X$ (под полным вложением здесь мы будем понимать вложение, сохраняющее решеточные пересечения конечных подмножеств и объединения произвольных подмножеств). Если решетка $L$ изоморфна решетке всех подмножеств множества $X$, то справедливо более сильное утверждение: условие (6) выполняется тогда и только тогда, когда $b$ является линейной комбинацией (в обычном, а не только в потенциальном смысле) строк $a^{(\gamma)}$.

Возвращаясь к примеру 1, покажем, что он на самом деле имеет весьма общий характер. Элемент $p$ в этом примере не имел дополнения. Покажем, что необходимым условием 
эквивалентности условия (6) и условия $b \in \operatorname{Span}\left\{a^{(\gamma)} \mid \gamma \in \Gamma\right\}$ является наличие в решетке $L$ относительных дополнений.

Напомним, что решетка $L$ называется решеткой с относительными дополнениями, если для любого интервала $[x, y]$ этой решетки для каждого элемента $p \in[x, y]$ существует такое $q \in[x, y]$, что $p \wedge q=x, p \vee q=y$.

Пусть $L-$ дистрибутивная решетка, в которой существуют точные верхние грани любых подмножеств и выполнено условие (4). Будем говорить, что решетка $L$ обладает свойством $(A)$, если для любых строк $b, a^{(\gamma)}, \gamma \in \Gamma$, условие (6) влечет справедливость включения $b \in \operatorname{Span}\left\{a^{(\gamma)} \mid \gamma \in \Gamma\right\}$.

Предложение 3. Если решетка L обладает свойством (A), то L-решетка с относительными дополнениями.

Доказательство. Пусть $x<y$ и $x \leqslant p \leqslant y$. Положим $a^{(1)}=(p, x), a^{(2)}=(y, p)$, $b=(y, x)$. Проверим, что

$$
\left(a^{(1)} \rho=a^{(1)} \rho^{\prime} \& a^{(2)} \rho=a^{(2)} \rho^{\prime}\right) \Longrightarrow b \rho=b \rho^{\prime} .
$$

Вложим решетку $L$ в какую-нибудь дистрибутивную решетку $\widetilde{L}$ с относительными дополнениями, например, в решетку $2^{X}$ (см. теорему 1 в разделе 4 гл. 2 в [5]). Пусть $q-$ дополнение элемента $p$ в интервале $[x, y]$ решетки $\widetilde{L}$. Нетрудно проверить, что

$$
(y, x)=(y \wedge(p, x)) \vee(q \wedge(y, p)),
$$

поэтому строка $b$ является потенциальной линейной комбинацией строк $a^{(1)}, a^{(2)}$. Из предложения 2 следует, что для этих строк выполнено условие (6). Решетка $L$ обладает свойством $(A)$, поэтому

$$
(y, x)=(\xi \wedge(p, x)) \vee(\eta \wedge(y, p))
$$

для некоторых $\xi, \eta \in L$.

Отсюда получаем, что

$$
y=(\xi \wedge p) \vee(\eta \wedge y), \quad x=(\xi \wedge x) \vee(\eta \wedge p) .
$$

Положив $\xi^{\prime}=\xi \wedge y, \eta^{\prime}=\eta \wedge y$, получим, что

$$
y=\left(\xi^{\prime} \wedge p\right) \vee\left(\eta^{\prime} \wedge y\right), \quad x=\left(\xi^{\prime} \wedge x\right) \vee\left(\eta^{\prime} \wedge p\right) .
$$

Далее положим $\xi^{\prime \prime}=\xi^{\prime} \vee x, \eta^{\prime \prime}=\eta^{\prime} \vee x$. Тогда

$$
y=\left(\xi^{\prime \prime} \wedge p\right) \vee\left(\eta^{\prime \prime} \wedge y\right), \quad x=\left(\xi^{\prime \prime} \wedge x\right) \vee\left(\eta^{\prime \prime} \wedge p\right) .
$$

Очевидно, $\xi^{\prime \prime}, \eta^{\prime \prime} \in[x, y]$. Поэтому

$$
y=\left(\xi^{\prime \prime} \wedge p\right) \vee \eta^{\prime \prime}, \quad x=x \vee\left(\eta^{\prime \prime} \wedge p\right) .
$$

Отсюда получаем, что $p \vee \eta^{\prime \prime} \geqslant\left(\xi^{\prime \prime} \wedge p\right) \vee \eta^{\prime \prime}=y$, поэтому $p \vee \eta^{\prime \prime}=y$. Кроме того, $\eta^{\prime \prime} \wedge p \leqslant x$, поэтому $p \wedge \eta^{\prime \prime}=x$. Следовательно, элемент $\eta^{\prime \prime} \in L$ является дополнением элемента $p$ в интервале $[x, y]$ решетки $L$.

Следствие 1. Конечная дистрибутивная решетка L обладает свойством $(A)$ в том и только в том случае, если L изоморфна решетке всех подмножеств некоторого множества. 
Лемма 3. Если решетки $L_{v}, v \in N$, с нулем обладают свойством (A), то их прямое произведение

$$
L=\prod_{v \in N} L_{v}
$$

также обладает свойством $(A)$.

Доказательство. Утверждение леммы непосредственно следует из того факта, что операции в решетке $\prod_{v \in N} L_{v}$ осуществляются покомпонентно. Действительно, пусть строки $a^{(\gamma)}, \gamma \in \Gamma$, и $b$ с элементами из $L$ таковы, что $\left(\forall \gamma a^{\gamma} \rho=a^{\gamma} \rho^{\prime}\right) \Longrightarrow b \rho=b \rho^{\prime}$ для любых столбцов $\rho$ и $\rho^{\prime}$. Зафиксируем $v_{0} \in N$. Пусть $\delta, \delta^{\prime}-$ столбцы с элементами из $L_{v_{0}}$ такие, что $a_{\nu_{0}}^{(\gamma)} \delta=a_{\nu_{0}}^{(\gamma)} \delta^{\prime}$ для всех $\gamma$. Положим

$$
\rho_{v}=\left\{\begin{array}{ll}
\delta, & \text { если } v=v_{0}, \\
0, & \text { если } v \neq v_{0},
\end{array} \quad \rho_{v}^{\prime}= \begin{cases}\delta^{\prime}, & \text { если } v=v_{0}, \\
0, & \text { если } v \neq v_{0} .\end{cases}\right.
$$

Тогда $a^{(\gamma)} \rho=a^{(\gamma)} \rho^{\prime}$. Отсюда следует, что $b \rho=b \rho^{\prime}$. Это влечет равенство $b_{v_{0}} \delta=b_{v_{0}} \delta^{\prime}$. Решетка $L_{v_{0}}$ обладает свойством $(A)$, поэтому

$$
b_{v_{0}}=\bigvee_{\gamma}\left(\lambda_{v_{0}}^{(\gamma)} \wedge a_{v_{0}}^{(\gamma)}\right)
$$

при некоторых $\lambda_{v_{0}}^{(\gamma)} \in L_{v_{0}}$. Составим из коэффициентов $\lambda_{v_{0}}^{(\gamma)}, v_{0} \in N$, элемент $\lambda^{(\gamma)} \in L$. Тогда получим, что

$$
b=\bigvee_{\gamma}\left(\lambda^{(\gamma)} \wedge a^{(\gamma)}\right) .
$$

Таким образом, $L$ обладает свойством $(A)$.

Лемма 4. Двухэлементная решетка $L=\{0,1\}$ обладает свойством $(A)$.

Доказательство. Предположим, что строки $b=\left\|b_{i}\right\|_{i \in I}$ и $a^{(\gamma)}=\left\|a_{i}^{(\gamma)}\right\|_{i \in I}, \gamma \in \Gamma$, из 0 и 1 удовлетворяют условию (6). Надо доказать, что $b \in \operatorname{Span}\left\{a^{(\gamma)} \mid \gamma \in \Gamma\right\}$. Лемма будет доказана, если мы покажем, что $b=\bigvee\left\{a^{(\gamma)} \mid a^{(\gamma)} \leqslant b\right\}$. Предположим обратное, что $b \neq \bigvee\left\{a^{(\gamma)} \mid a^{(\gamma)} \leqslant b\right\}$. Тогда найдется $i_{0} \in I$ такое, что $\bigvee\left\{a_{i_{0}}^{(\gamma)} \mid a^{(\gamma)} \leqslant b\right\}<b_{i_{0}}$. Это означает, что $b_{i_{0}}=1, \mathrm{a} \bigvee\left\{a_{i_{0}}^{(\gamma)} \mid a^{(\gamma)} \leqslant b\right\}=0$. Положим $\Gamma_{1}=\left\{\gamma \in \Gamma \mid a^{(\gamma)} \leqslant b\right\}$, $\Gamma_{2}=\Gamma \backslash \Gamma_{1}$. Очевидно, что $a_{i_{0}}^{(\gamma)}=0$ при $\gamma \in \Gamma_{1}$. Положим $\rho_{i}=1-b_{i}$ при всех $i \in I$,

$$
\rho_{i}^{\prime}= \begin{cases}\rho_{i} & \text { при } i \neq i_{0}, \\ 1 & \text { при } i \neq i_{0} .\end{cases}
$$

Проверим, что $a^{(\gamma)} \rho=a^{(\gamma)} \rho^{\prime}$. Пусть вначале $\gamma \in \Gamma_{1}$. Тогда $a^{(\gamma)} \leqslant b$ и, следовательно, $a^{(\gamma)} \rho \leqslant b \rho=\bigvee_{i} b_{i}\left(1-b_{i}\right)=0$. Кроме того, $a^{(\gamma)} \rho^{\prime}=\bigvee_{i} a_{i}^{(\gamma)} \rho_{i} \vee a_{i_{0}}^{(\gamma)} \rho_{i_{0}}=0 \vee 0=0$. Пусть теперь $\gamma \in \Gamma_{2}$. Тогда $a^{(\gamma)} \not \leq b$, поэтому найдется такое $i \in I$, что $a_{i}^{(\gamma)}=1, b_{i}=0$. Справедливы соотношения $a^{(\gamma)} \rho \geqslant a_{i}^{(\gamma)} \rho_{i}=1, a^{(\gamma)} \rho^{\prime} \geqslant a^{(\gamma)} \rho=1$. Следовательно, $a^{(\gamma)} \rho=a^{(\gamma)} \rho^{\prime}=1$. Итак, $a^{(\gamma)} \rho=a^{(\gamma)} \rho^{\prime}$ при всех $\gamma \in \Gamma$. Вместе с тем справедливы равенства $b \rho=\bigvee_{i} b_{i}\left(1-b_{i}\right)=0, b \rho^{\prime} \geqslant b_{i_{0}} \rho_{i_{0}}^{\prime}=1$, то есть $b \rho \neq b \rho^{\prime}$. Мы видим, что условие (6) не выполнено. 
Предложение 4. Решетка $2^{X}$ всех подмножеств множества X обладает свойством $(A)$.

Доказательство. Хорошо известно, что решетка $2^{X}$ является прямым произведением $|X|$ решеток $\{0,1\}$. Поэтому предложение следует из лемм 3,4 .

Вернемся теперь к матрицам над дистрибутивными решетками. Как и ранее, мы будем предполагать решетки дистрибутивными, имеющими супремум любых подмножеств и удовлетворяющими условию (4). Пусть $\sigma=\left\|\sigma_{k j}\right\|_{k \in K}, j \in J$, и $\tau=\left\|\tau_{i j}\right\|_{i \in I, j \in J},-$ матрицы над дистрибутивной решеткой $L$, которая удовлетворяет вышеприведенным требованиям. Будем говорить, что $\tau$ делится (слева) на $\sigma$, и писать $\tau \leqslant l ~ \sigma$, если существует такая $I \times K$ матрица $\mu$ над $L$, что $\tau=\mu \sigma$.

Теорема 3. Пусть $L-$-решетка всех подмножеств некоторого множества $X$, $\sigma$ и $\tau-$ $K \times J$ и $I \times J$ матрицы над L. Тогда $\tau \leqslant_{l} \sigma$ в том и только в том случае, если

$$
\forall \rho, \rho^{\prime} \quad\left(\sigma \rho=\sigma \rho^{\prime} \Longrightarrow \tau \rho=\tau \rho^{\prime}\right),
$$

где $\rho=\left\|\rho_{j}\right\|_{j \in J}$ и $\rho^{\prime}=\left\|\rho_{j}^{\prime}\right\|_{j \in J}-$ столбцьь.

Доказательство. Если $\tau=\mu \sigma$, то условие (8), очевидно, выполняется. Обратно, пусть выполнено (8). Пусть $b=\left\|\tau_{i j}\right\|_{j \in J}-i$-я строка матрицы $\tau, a^{(k)}=\left\|\sigma_{k j}\right\|_{j \in J}, k \in K,-$ строки матрицы $\sigma$. Из (8) следует, что $\left(\forall k a^{(k)} \rho=a^{(k)} \rho^{\prime}\right) \Longrightarrow b \rho=b \rho^{\prime}$. По предложению $4, b \in \operatorname{Span}\left\{a^{(k)} \mid k \in K\right\}$. Все строки матрицы $\tau$ линейно выражаются через строки матрицы $\sigma$, поэтому $\tau=\mu \sigma$.

Обозначим через $B_{I}(L)$ множество квадратных $I \times I$ матриц над полной дистрибутивной решеткой $L$. Очевидно, что $B_{I}(L)-$ полугруппа.

Предложение 5. Если $L-$ решетка всех подмножеств некоторого множества $X$, a I - произвольное множество, то в полугруппе $B_{I}(L)$ имеют место равенства $\leqslant *=\leqslant$, $\mathscr{L}^{*}=\mathscr{L}$.

Доказательство. Ясно, что достаточно доказать равенство $\leqslant_{l}^{*}=\leqslant_{l}$. Для этого достаточно установить, что $\leqslant_{l}^{*} \subseteq \leqslant_{l}$. Пусть $\sigma, \tau \in B_{I}(L)$ таковы, что $\tau \leqslant *$. Тогда $\sigma \beta=\sigma \beta^{\prime} \Longrightarrow \tau \beta=\tau \beta^{\prime}$ для любых $\beta, \beta^{\prime} \in B_{I}(L)$. Отсюда $\sigma \rho=\sigma \rho^{\prime} \Longrightarrow \tau \rho=\tau \rho^{\prime}$ для любых столбцов $\rho, \rho^{\prime}$, и (8) выполнено. Из теоремы 3 следует, что $\tau \leqslant l ~ \sigma$.

Рассмотрим следующее условие $(B)$ на решетку $L: L-$ полная дистрибутивная решетка, имеющая полное вложение $L \rightarrow \widetilde{L}$ в решетку $\widetilde{L}$ всех подмножеств некоторого множества $X$, при котором $0 \mapsto 0,1 \mapsto 1$; будем отождествлять решетку $L$ с ее образом при этом вложении, то есть считать, что $L \subseteq \widetilde{L}$.

Теорема 4. Пусть $L$ - решетка, удовлетворяющая условию (B), I - произвольное множество, $B_{I}(L)-$ полугруппа $I \times I$ матрии над $L u \sigma, \tau \in B_{I}(L)$. Тогда эквивалентны следующие условия:

(i) $\sigma \rho=\sigma \rho^{\prime} \Longrightarrow \tau \rho=\tau \rho^{\prime}$ для любых столбиов $\rho$, $\rho^{\prime}$ размера $I \times 1$;

(ii) $\sigma \beta=\sigma \beta^{\prime} \Longrightarrow \tau \beta=\tau \beta^{\prime}$ для любых матрии $\beta, \beta^{\prime} \in B_{I}(L)$;

(iii) $\tau=\mu \sigma$ для некоторой матрищьы $\mu \in B_{I}(\widetilde{L})$. 
Доказательство. Импликация (i) $\Rightarrow$ (ii) следует из определения матричного умножения. Импликация (iii) $\Rightarrow$ (i) очевидна. Докажем эквивалентность (ii) $\Longleftrightarrow$ (iii). Пусть выполнено (ii). Тогда $\tau \leqslant *$ в в полугруппе $B_{I}(L)$. Так как $B_{I}(L)-$ подполугруппа полугруппы $B_{I}(\tilde{L})$, а в полугруппе $B_{I}(\widetilde{L})$ ввиду предложения $5 \leqslant_{l}^{*}=\leqslant_{l}$, по теореме $1 \tau=\mu \sigma$ для некоторой матрицы $\mu \in B_{I}(\widetilde{L})$.

Предложение 6. Пусть $L-$ удовлетворяющяая условию (В) решетка, $b=\left\|b_{j}\right\|_{j \in J} u$ $a^{(k)}=\left\|a_{j}^{(k)}\right\|_{j \in J}, k \in K,-$ строки с элементами из L. Тогда эквивалентнь следующие условия:

(i) $\left(\forall k a^{(k)} \rho=a^{(k)} \rho^{\prime}\right) \Longrightarrow b \rho=b \rho^{\prime}$ для любых столбиов $\rho, \rho^{\prime}$ размера $J \times 1$;

(ii) $b \in \operatorname{Span}\left\{a^{(k)} \mid k \in K\right\}$, если строки $a^{(k)}(k \in K)$ и b рассматривать как строки с элементами из $\widetilde{L}$.

Доказательство. Импликация (ii) $\Rightarrow$ (i) очевидна. Докажем импликацию (i) $\Rightarrow$ (ii). Рассмотрим матрицу $\sigma=\left\|a_{j}^{(k)}\right\|_{k \in K, j \in J}$. Если $|K|<|J|$, то добавим к матрице $\sigma$ нулевые строки так, чтобы получилась квадратная матрица $\hat{\sigma}$; если $|K|>|J|$, то удлиним строки матрицы $\sigma$, добавив в эти строки справа нулевые элементы для получения квадратной матрицы $\hat{\sigma}$; если $|K|=|J|$, то полагаем $\hat{\sigma}=\sigma$. Далее, матрица $\hat{\tau}-$ это матрица, у которой все строки равны $b$, если $|K| \leqslant|J|$, и все строки равны удлиненной строке $b$, если $|K|>|J|$. Мы получили матрицы $\hat{\sigma}, \hat{\tau} \in B_{M}(L)$, где $M$ равно $K$ или $J$.

Пусть выполнено условие (i). Тогда $\hat{\sigma}=\hat{\sigma} \beta^{\prime} \Longrightarrow \hat{\tau} \beta=\hat{\tau} \beta^{\prime}$ при всех $\beta, \beta^{\prime} \in B_{M}(L)$. Используя теорему 4 , получаем, что $\hat{\tau}=\hat{\mu} \hat{\sigma}$ для некоторой матрицы $\hat{\mu} \in B_{M}(\tilde{L})$. Отсюда следует, что $b \in \operatorname{Span}\left\{a^{(k)} \mid k \in K\right\}$ над решеткой $\widetilde{L}$.

Теорема 5. Пусть $L-$ - решетка, удовлетворяюшая условию $(B), \sigma u \tau-K \times J u I \times J$ матрицы над L. Тогда эквивалентны следующие условия:

(i) $\sigma \rho=\sigma \rho^{\prime} \Longrightarrow \tau \rho=\tau \rho^{\prime}$ для любых столбиов $\rho, \rho^{\prime}$ размера $J \times 1$ над L;

(ii) $\tau=\mu \sigma$ для некоторой $K \times I$ матриц̧ы $\mu$ над решеткой $\widetilde{L}$.

Доказательство. Импликация (ii) $\Rightarrow$ (i) очевидна. Докажем обратную импликацию. Пусть выполнено условие (i). Тогда по предложению 6 каждая строка матрицы $\tau$ является линейной комбинацией строк матрицы $\sigma$ с коэффициентами из решетки $\widetilde{L}$. Следовательно, $\tau=\mu \sigma$ для некоторой матрицы $\mu$ над $\widetilde{L}$.

Разберем теперь подробно случай матриц конечного размера над дистрибутивной решеткой $L$. Теперь никаких требований на решетку $L$, кроме дистрибутивности, не налагается.

Пусть $L-$ дистрибутивная решетка, $b=\left(b_{1}, \ldots, b_{n}\right)$ и $a^{(i)}=\left(a_{1}^{(i)}, \ldots, a_{n}^{(i)}\right)$, $i=1,2, \ldots, m,-$ строки с элементами из $L$. Рассмотрим следующее условие на эти строки, являющееся частным случаем условия (6): для всех столбцов $\rho=\left(\rho_{1}, \ldots, \rho_{n}\right)$, $\rho^{\prime}=\left(\rho_{1}^{\prime}, \ldots, \rho_{n}^{\prime}\right)$ с элементами из $L$

$$
\left(\forall i a^{(i)} \rho=a^{(i)} \rho^{\prime}\right) \Longrightarrow b \rho=b \rho^{\prime} .
$$

Предложение 7. Если условие (9) при некотором $n \geqslant 2$ влечет условие $b \in \operatorname{Span}\left(a^{(1)}, \ldots, a^{(m)}\right)$ для всех строк длинь $n$, то $L-$ решетка с относительньми дополнениями. Обратно, если $L-$ решетка с относительными дополнениями, то для любого $n \geqslant 1$ и любых строк $b, a^{(i)}, \ldots, a^{(m)}$ длины $n$ условие (9) влечет условие $b \in \operatorname{Span}\left(a^{(1)}, \ldots, a^{(m)}\right)$. 
Доказательство. Первая часть предложения доказывается так же, как предложение 3. Докажем вторую часть. Пусть $b, a^{(1)}, \ldots, a^{(m)}-$ строки, удовлетворяющие условию (9). Обозначим через $L_{0}$ решетку, порожденную всеми элементами строк $b, a^{(1)}, \ldots, a^{(m)}$. Тогда $L_{0}-$ конечная решетка. Пусть $\theta-$ наименьший, а $\omega-$ наибольший элемент решетки $L_{0}$. Обозначим через $L_{1}$ решетку, порожденную элементами решетки $L_{0}$ и их дополнениями в интервале $[\theta, \omega]$. Тогда $L_{1}-$ конечная булева алгебра, поэтому $L_{1}$ изоморфна решетке всех подмножеств конечного множества. Так как условие (9) выполняется для всех столбцов с элементами из $L$, оно выполняется и для столбцов с элементами из $L_{1}$. Теперь из предложение 4 следует, что $b \in \operatorname{Span}\left(a^{(1)}, \ldots, a^{(m)}\right)$.

Замечание 2. Так как условие $b \in \operatorname{Span}\left(a^{(1)}, \ldots, a^{(m)}\right)$ влечет условие (9) для любой решетки, в формулировке предложения 7 слово влечет можно заменить словом эквивалентно.

Предложение 8. Пусть $L-$ произвольная дистрибутивная решетка, $\sigma u \tau-p \times n u$ $m \times n$ матрицы над L. Тогда эквивалентны следующие условия:

(i) $\sigma \rho=\sigma \rho^{\prime} \Longrightarrow \tau \rho=\tau \rho^{\prime}$ для любых столбичов длины $n$ над $L$;

(ii) $\sigma \beta=\sigma \beta^{\prime} \Longrightarrow \tau \beta=\tau \beta^{\prime}$ для любых матрии $n \times t$ над $L$;

(iii) $\underset{\tau}{\tau}=\mu \sigma$ для некоторой $m \times p$ матрицы с элементами из некоторой надрешетки $\tilde{L} \supseteq L$.

Доказательство. Эквивалентность (i) $\Longleftrightarrow$ (ii) следует из определения матричного умножения. Импликация (iii) $\Rightarrow$ (i) очевидна. Докажем импликацию (i) $\Rightarrow$ (iii). Вложим решетку $L$ в какую-нибудь булеву алгебру $\widetilde{L}$ (например, в решетку всех подмножеств множества). Обозначим через $L_{1}$ булеву алгебру, порожденную в $\widetilde{L}$ всеми элементами матриц $\sigma$ и $\tau$. Очевидно, условие (i) будет выполняться для любых столбцов $\rho, \rho^{\prime}$ с элементами из $L_{1}$. Так как $L_{1}-$ конечная булева алгебра, она изоморфна решетке всех подмножеств конечного множества. В силу теоремы 3 тогда $\tau=\mu \sigma$ для некоторой $m \times p$ матрицы над $L_{1}$. Элементы матрицы $\mu$ принадлежат $L_{1} \subseteq \widetilde{L}$.

Заметим, что если $L-$ конечная решетка, то решетка $\tilde{L}$ в предложении 8 может быть также выбрана конечной.

Полугруппу матриц размера $n \times n$ над дистрибутивной решеткой $L$ будем обозначать $B_{n}(L)$.

Предложение 9. Пусть $L-$ дистрибутивная решетка, $n$ - натуральное число $u$ $\sigma, \tau \in B_{n}(L)$. Тогда $\tau \leqslant \leqslant_{l}^{*} \sigma$ в том и только том случае, если существует такая надрешетка $\tilde{L} \supseteq L$ и матриия $\mu \in B_{n}(\widetilde{L})$ такая, что $\tau=\mu \sigma$.

Доказательство проводится аналогично доказательству теоремы 4.

Заметим, что если $L-$ конечная решетка, то решетку $\widetilde{L}$ в предложении 9 также можно считать конечной.

Теорема 6. Пусть $L-$ дистрибутивная решетка и $n \geqslant 2-$ натуральное число. Тогда эквивалентны следующие условия:

(i) L-реметка с относительными дополнениями;

(ii) в полугруппе $B_{n}(L)$ совпадают отномения $\leqslant_{l}^{*} u \leqslant_{l}$; 
(iii) в полугруппе $B_{n}(L)$ справедливо равенство $\mathscr{L}^{*}=\mathscr{L}$.

Доказательство. Докажем импликацию (i) $\Rightarrow$ (ii). Пусть $L-$ решетка с относительными дополнениями и $\tau \leqslant \leqslant_{l}^{*} \sigma$. Обозначим через $L_{1}$ конечную булеву алгебру, порожденную всеми элементами матриц $\sigma$ и $\tau$. Так как $\tau \leqslant \leqslant_{l}^{*} \sigma$, выполнено условие (8). Отсюда по теореме $3 \tau \leqslant_{l} \sigma$. Импликация (ii) $\Rightarrow$ (iii) очевидна. Докажем импликацию (iii) $\Rightarrow$ (i). Пусть $L$ - дистрибутивная решетка, не все элементы которой имеют относительные дополнения. Для простоты будем считать, что $0,1 \in L$ и элемент $p \in L$ не имеет дополнения. Проверим, что для матриц $\sigma=\left(\begin{array}{ll}1 & 0 \\ 1 & p\end{array}\right)$ и $\tau=\left(\begin{array}{ll}p & 0 \\ 1 & p\end{array}\right)$ выполняются соотношения $(\sigma, \tau) \in \mathscr{L}^{*}$, $(\sigma, \tau) \notin \mathscr{L}$. Действительно, в примере 1 было показано, что строка $(1,0)$ матрицы $\sigma$ не является линейной комбинацией строк $(p, 0)$ и $(1, p)$ матрицы $\tau$, поэтому $(\sigma, \tau) \notin \mathscr{L}$. Вместе с тем, если погрузить $L$ в дистрибутивную решетку, в которой элемент $p$ имеет дополнение $q$, то равенства

$$
\left(\begin{array}{ll}
1 & 0 \\
1 & p
\end{array}\right)=\left(\begin{array}{ll}
1 & q \\
0 & 1
\end{array}\right)\left(\begin{array}{ll}
p & 0 \\
1 & p
\end{array}\right), \quad\left(\begin{array}{ll}
p & 0 \\
1 & p
\end{array}\right)=\left(\begin{array}{ll}
p & 0 \\
0 & 1
\end{array}\right)\left(\begin{array}{ll}
1 & 0 \\
1 & p
\end{array}\right)
$$

покажут, что $(\sigma, \tau) \in \mathscr{L}^{*}$. Небольшим усложнением этого примера можно построить такие матрицы $\sigma$ и $\tau$ в любом интервале $[x, y]$ решетки $L$, если там есть элемент, не имеющий относительного дополнения, причем по $2 \times 2$ матрицам можно построить матрицы любого размера, принадлежащие $\mathscr{L}^{*} \backslash \mathscr{L}$. Таким образом, существование относительных дополнений является и необходимым условием равенства $\mathscr{L}^{*}=\mathscr{L}$.

Заметим, что утверждение, аналогичное теореме 6, справедливо и для двойственных отношений $\leqslant_{r}, \leqslant_{r}^{*}, \mathscr{R}, \Re^{*}$.

Следствие 2. В полугруппе $B_{n}(\{0,1\})$ всех $n \times n$ матрии из 0 и 1 справедливы равенства $\leqslant_{l}^{*}=\leqslant_{l}, \mathscr{L}^{*}=\mathscr{L}$, а значит, и двойственные к ним равенства $\leqslant_{r}^{*}=\leqslant_{r}, \mathscr{R}^{*}=\mathscr{R}$.

В заключение отметим, что в нашей работе рассматривалась только делимость матриц над решетками друг на друга слева или справа. Мы ничего не можем сказать о двусторонней делимости (то есть о представлении $\tau$ в виде $\tau=\alpha \sigma \beta$ ). Распознавание двусторонней делимости даже для матриц конечного размера из 0 и 1 является $N P$-полной задачей (см. [9]).

\section{Список литературы}

1. Ляпин Е. С., Полугруппы. Физматлит, Москва, 1960.

2. Шутов Э. Г., Потенциальная делимость элементов в полугруппах. Уч. зап. Ленингр. гос. пед. ин-та им. Гериена (1958) 166, 75-103.

3. Clark C. E., Carruth J. H., Generalized Green's theories. Semigroup Forum (1980) 20, 95-127.

4. Клиффорд А., Престон Г., Алгебраическая теория полугрупп. Мир, Москва, 1972.

5. Гретцер Г., Общзая теория решеток. Наука, Москва, 1982.

6. Kim K. H., Boolean matrix theory and applications. Marcel Dekker, New York, 1982.

7. Поплавский В. Б., О рангах, классах Грина и теории определителей булевых матриц. Дискретная математика (2008) 20, №4, 42-60.

8. Fountain J. B., Abundant semigroups. Proc. London Math. Soc. (1982) 44, №3, 103-129.

9. Markowsky G., Ordering D-classes and computing Schein rank is hard. Semigroup Forum (1992) 44, №3, 373-375. 